\title{
Pion light-front wave function, parton distribution and the electromagnetic form factor
}

\author{
Thomas Gutsche ${ }^{1}$, Valery E. Lyubovitskij ${ }^{1,2,3}$, Ivan Schmidt ${ }^{4}$, \\ Alfredo Vega ${ }^{5,6}$
}

${ }^{1}$ Institut für Theoretische Physik, Universität Tübingen, Kepler Center for Astro and Particle Physics, Auf der Morgenstelle 14, D-72076 Tübingen, Germany

${ }^{2}$ Department of Physics, Tomsk State University, 634050 Tomsk, Russia

${ }^{3}$ Mathematical Physics Department, Tomsk Polytechnic University, Lenin avenue 30, 634050 Tomsk, Russia

${ }^{4}$ Departamento de Física y Centro Científico Tecnológico de Valparaíso (CCTVal), Universidad Técnica Federico Santa María, Casilla 110-V, Valparaíso, Chile

${ }^{5}$ Instituto de Física y Astronomía, Universidad de Valparaíso, Avenida Gran Bretaña 1111, Valparaíso, Chile

${ }^{6}$ Centro de Astrofísica de Valparaíso, Avenida Gran Bretaña 1111, Valparaíso, Chile

E-mail: thomas.gutsche@uni-tuebingen.de, valeri.lyubovitskij@uni-tuebingen.de, ivan.schmidt@usm.cl, alfredo.vega@uv.cl

\begin{abstract}
.
We derive the lowest-order $q \bar{q}$ valence light-front wave function for the pion with $L_{z}=0$ and $\left|L_{z}\right|=1$, which reproduces its valence parton distribution and the electromagnetic form factor consistent with data.
\end{abstract}

PACS: 12.38.Lg, 12.39.Ki, 13.40.Gp, 14.40.Be

Keywords: pion, light-front quark model, parton distribution, electromagnetic form factor 
Pion light-front wave function, parton distribution and the electromagnetic form factor 2

\section{Introduction}

The main purpose of this paper is to derive the lowest-order $q \bar{q}$ valence light-front wave function (LFWF) for the pion with $L_{z}=0$ and $\left|L_{z}\right|=1$, which can generate a pion parton distribution function $(\mathrm{PDF}) q_{\pi}(x)$ and the electromagnetic form factor $F_{\pi}\left(Q^{2}\right)$ consistent with data. The present study is based on data on the pion electromagnetic form factor [1]-[6] and the model-independent result for the pion PDF at the initial scale $\mu_{0}=0.63 \mathrm{GeV}$

$$
x q_{\pi}\left(x, \mu_{0}\right)=N_{\pi} x^{\alpha}(1-x)^{\beta}\left(1+\gamma x^{\delta}\right)
$$

derived in Ref. [7]. The result of [7] rests on an updated analysis of the E615 data on the cross section of the Drell-Yan (DY) process $\pi^{-} N \rightarrow \mu^{+} \mu^{-} X$ [8] including next-toleading logarithmic (NLL) threshold resummation effects. The coefficients $\alpha, \beta, \gamma$ and $\delta$ are free parameters, which for the best fit of Ref. [7] are reported as

$$
\alpha=0.70, \beta=2.03, \gamma=13.8, \delta=2 \text {. }
$$

The normalization constant $N_{\pi}$ is fixed by the condition

$$
\int_{0}^{1} d x q_{\pi}\left(x, \mu_{0}\right)=1 .
$$

These parameters were fixed by evolving the pion PDF to the higher scale $\mu=4 \mathrm{GeV}$, where the E615 data were extracted and analyzed leading to

$$
q_{\pi}(x, \mu=4 \mathrm{GeV}) \sim(1-x)^{2.34}
$$

for large $x$. The main result of Ref. [7] is that the pion PDF at the initial scale and for large $x$ with

$$
q_{\pi}\left(x, \mu_{0}\right) \sim(1-x)^{2.03}
$$

is considerably softer when compared to the next-to-leading order analysis of the DY process performed previously (see e.g. Refs. [9]-[11]). Note that the result of Ref. [7] is in agreement with calculations based on the Dyson-Schwinger approach [12]. The pion PDF has also been subject of detailed analyses in quark models, as for example the one performed in the chiral quark model [13]. As in the series of NLO analysis that have been made without the inclusion of soft-gluon resummation, the analysis of Ref. [13] was based on a harder PDF at the scale $\mu=4 \mathrm{GeV}$ with

$$
q_{\pi}(x, \mu=4 \mathrm{GeV}) \sim(1-x) .
$$

Using the prediction of the chiral quark model for the pion PDF at the initial scale with $q_{\pi}\left(x, \mu_{0}\right)=1$, this initial scale $\mu_{0} \simeq 313 \mathrm{MeV}$ was determined by evolving the $q_{\pi}(x, \mu)$ down from $\mu=4 \mathrm{GeV}$. Recently [14] we could confirm the results of Ref. [13]. In particular, we applied a soft-wall AdS/QCD model [15]-[20] for the analysis of the evolution of the pion PDF. As in Ref. [13], the pion PDF obtained in soft-wall AdS/QCD 
Pion light-front wave function, parton distribution and the electromagnetic form factor3

should be considered at the initial scale. At $\mu=4 \mathrm{GeV}$ the result of [14] coincides with the result of the chiral quark model [13] and is very well approximated by the form

$$
q_{\pi}(x, \mu=4 \mathrm{GeV})=1.108 x^{-0.381}(1-x)^{1.323} .
$$

A nice feature of the soft-wall AdS/QCD approach is that it allows for an extraction of the hadronic LFWF. The idea to extract LFWFs from AdS/QCD was originally suggested in Ref. [15] considering the pion electromagnetic form factor in two approaches - AdS/QCD and light-front QCD. In a series of papers $[15,17,18,16,19]$ this problem was further discussed in detail. The pionic $L_{z}=S_{z}=0$ LFWF extracted from AdS/QCD reads [16]

$$
\psi_{\pi}^{\operatorname{AdS}}\left(x, \mathbf{k}_{\perp}\right)=\frac{4 \pi}{\kappa} \frac{\sqrt{\log (1 / x)}}{1-x} \exp \left[-\frac{\mathbf{k}_{\perp}^{2}}{2 \kappa^{2}} \frac{\log (1 / x)}{(1-x)^{2}}\right]
$$

where $\kappa=350 \mathrm{MeV}$ is the dilaton scale parameter [14]. Note that the derived LFWF is not symmetric under the exchange $x \rightarrow 1-x$, since it results from the matching of the matrix element for the bare electromagnetic current between the dressed LFWF in LF QCD and the dressed electromagnetic current between hadronic wave functions in AdS/QCD. The recent analysis of Ref. [7] shows, however, that inclusion of the NLL threshold resummation effects in the $\pi^{-} N \rightarrow \mu^{+} \mu^{-} X$ cross section leads to a considerably softer pion PDF at the initial (low) scale. This obviously calls for a reconsideration of the pionic LFWF.

\section{Light-front wave function of the pion and applications}

In this section we offer an updated pion LFWF which, first, produces the PDF extracted in the analysis of [7] and, second, results in a pion electromagnetic form factor consistent with current data. Following Ref. [21, 22, 23] we construct the lowest quarkantiquark Fock component LFWF for the pion, which includes the superposition of three components with the total quark orbital angular momentum $L_{z}=0, \pm 1$. Considering for example a positively charged pion with momentum $P=\left(P^{+},\left(M_{\pi}^{2}+\mathbf{P}_{\perp}^{2}\right) / P^{+}, \mathbf{P}_{\perp}\right)$ the light-front pion state reads [21, 22, 23]

$$
\begin{aligned}
\left|\pi^{+}\left(P^{+}, \mathbf{P}_{\perp}\right)\right\rangle & =\left|\pi^{+}\left(P^{+}, \mathbf{P}_{\perp}\right)\right\rangle_{L_{z}=0}+\left|\pi^{+}\left(P^{+}, \mathbf{P}_{\perp}\right)\right\rangle_{\left|L_{z}\right|=1}, \\
\left|\pi^{+}\left(P^{+}, \mathbf{P}_{\perp}\right)\right\rangle_{L_{z}=0} & =\int \frac{d^{2} \mathbf{k}_{\perp}}{16 \pi^{3}} \frac{d x}{\sqrt{x(1-x)}} \psi_{\pi}^{(0)}\left(x, \mathbf{k}_{\perp}\right)\left|x, \mathbf{k}_{\perp} ; P^{+}, \mathbf{P}_{\perp}, L_{z}=0\right\rangle, \\
\left|\pi^{+}\left(P^{+}, \mathbf{P}_{\perp}\right)\right\rangle_{\left|L_{z}\right|=1} & =\int \frac{d^{2} \mathbf{k}_{\perp}}{16 \pi^{3}} \frac{d x}{\sqrt{x(1-x)}} \psi_{\pi}^{(1)}\left(x, \mathbf{k}_{\perp}\right) \\
& \times \frac{1}{\sqrt{2}}\left(k_{\perp}^{+}\left|x, \mathbf{k}_{\perp} ; P^{+}, \mathbf{P}_{\perp}, L_{z}=1\right\rangle+k_{\perp}^{-}\left|x, \mathbf{k}_{\perp} ; P^{+}, \mathbf{P}_{\perp}, L_{z}=-1\right\rangle\right),
\end{aligned}
$$

where $k_{\perp}^{ \pm}=k_{1} \pm i k_{2}, \psi_{\pi}^{(0)}\left(x, \mathbf{k}_{\perp}\right)$ and $\psi_{\pi}^{(1)}\left(x, \mathbf{k}_{\perp}\right)$ are the pion $L_{z}=0$ and $\left|L_{z}\right|=$ 1 phenomenological LFWFs, respectively; $\left.\left|x, \mathbf{k}_{\perp} ; P^{+}, \mathbf{P}_{\perp}, L_{z}\right\rangle\right)$ is the $u \bar{d}$ Fock state with total quark orbital angular momentum $L_{z}$. The Fock states $\left|\pi^{+}\left(P^{+}, \mathbf{P}_{\perp}\right)\right\rangle_{L_{z}}$, 
Pion light-front wave function, parton distribution and the electromagnetic form factor4 $\left|x, \mathbf{k}_{\perp} ; P^{+}, \mathbf{P}_{\perp}, L_{z}\right\rangle$ and LFWFs $\psi_{\pi}^{(i)}\left(x, \mathbf{k}_{\perp}\right)$ are normalized according to

$$
\begin{aligned}
\left\langle x^{\prime}, \mathbf{k}_{\perp}^{\prime} ; P^{+^{\prime}}, \mathbf{P}_{\perp}^{\prime}, L_{z}^{\prime} \mid x, \mathbf{k}_{\perp} ; P^{+}, \mathbf{P}_{\perp}, L_{z}\right\rangle & =2 P^{+} 2 x(1-x)\left(2 \pi^{3}\right)^{2} \delta\left(P^{+}-P^{+^{\prime}}\right) \delta\left(x-x^{\prime}\right) \\
& \times \delta^{2}\left(\mathbf{k}_{\perp}-\mathbf{k}_{\perp}^{\prime}\right) \delta^{2}\left(\mathbf{P}_{\perp}-\mathbf{P}_{\perp}^{\prime}\right) \delta_{L_{z} L_{z}^{\prime}}, \\
\left\langle\pi^{+}\left(P^{+^{\prime}}, \mathbf{P}_{\perp}^{\prime}\right) \mid \pi^{+}\left(P^{+}, \mathbf{P}_{\perp}\right)\right\rangle & =2 P^{+}(2 \pi)^{3} \delta\left(P^{+}-P^{+^{\prime}}\right) \delta^{2}\left(\mathbf{P}_{\perp}-\mathbf{P}_{\perp}^{\prime}\right)
\end{aligned}
$$

and

$$
\begin{aligned}
1 & =\int_{0}^{1} d x q_{\pi}(x), \\
q_{\pi}(x) & =\int \frac{d^{2} \mathbf{k}_{\perp}}{16 \pi^{3}}\left[\left|\psi_{\pi}^{(0)}\left(x, \mathbf{k}_{\perp}\right)\right|^{2}+\mathbf{k}_{\perp}^{2}\left|\psi_{\pi}^{(1)}\left(x, \mathbf{k}_{\perp}\right)\right|^{2}\right] .
\end{aligned}
$$

In last equation $q_{\pi}(x)$ is the pion valence parton distribution function (PDF). A representation of the $u \bar{d}$ Fock states $\left|x, \mathbf{k}_{\perp} ; P^{+}, \mathbf{P}_{\perp}, L_{z}\right\rangle$ in terms of creation operators of $u$ - and $\bar{d}$-quarks is given in Appendix A. We restrict to the isospin limit, therefore $q_{\pi}(x)$ coincides with the distribution of $u$ and $\bar{d}$ - quarks in the pion

$$
q_{\pi}(x)=u_{v}^{\pi}(x)=\bar{d}_{v}^{\pi}(x) .
$$

The pion electromagnetic form factor $F_{\pi}\left(Q^{2}\right)$ (see e.g. details in Ref. [15]) is defined as

$$
\left\langle\pi^{+}\left(P^{+}, \mathbf{q}_{\perp}\right)\left|J^{+}(0)\right| \pi^{+}\left(P^{+}, \mathbf{0}_{\perp}\right)\right\rangle=2 P^{+} F_{\pi}\left(Q^{2}\right)
$$

where we choose the light-front frame coordinates of the initial pion $(P)$ and photon $(q)$ as $[15]$

$$
P=\left(P^{+}, M_{\pi}^{2} / P^{+}, \mathbf{0}_{\perp}\right), \quad q=\left(0,2 q P / P^{+}, \mathbf{q}_{\perp}\right) .
$$

Here $J^{+}(0)=\sum_{q} e_{q} \bar{q}(0) \gamma^{+} q(0)$ is the electromagnetic current operator (see its representation in terms of creation and annihilation operators of quarks and antiquarks in Ref. [15] and also in Appendix A). Evaluating Eq. (13) gives the representation of the pion electromagnetic form factor in terms of the LFWFs $\psi_{\pi}^{(0)}\left(x, \mathbf{k}_{\perp}\right)$ and $\psi_{\pi}^{(1)}\left(x, \mathbf{k}_{\perp}\right)$ (Drell-Yan-West formula [25])

$$
\begin{aligned}
& F_{\pi}\left(Q^{2}\right)=\int_{0}^{1} d x \mathcal{H}_{\pi}\left(x, Q^{2}\right), \\
& \mathcal{H}_{\pi}\left(x, Q^{2}\right)=\int \frac{d^{2} \mathbf{k}_{\perp}}{16 \pi^{3}}\left[\psi_{\pi}^{(0) \dagger}\left(x, \mathbf{k}_{\perp}^{\prime}\right) \psi_{\pi}^{(0)}\left(x, \mathbf{k}_{\perp}\right)+\mathbf{k}_{\perp}^{\prime} \mathbf{k}_{\perp} \psi_{\pi}^{(1) \dagger}\left(x, \mathbf{k}_{\perp}^{\prime}\right) \psi_{\pi}^{(1)}\left(x, \mathbf{k}_{\perp}\right)\right]
\end{aligned}
$$

where $\mathbf{k}_{\perp}^{\prime}=\mathbf{k}_{\perp}+(1-x) \mathbf{q}_{\perp}$ and $Q^{2}=\mathbf{q}_{\perp}^{2}$ and

$$
\mathcal{H}_{\pi}\left(x, Q^{2}\right)=H_{\pi}\left(x, \xi=0, Q^{2}\right)-H_{\pi}\left(-x, \xi=0, Q^{2}\right)
$$

is the nonforward parton density (NPD) [24] for the pion evaluated at zero skewness $\xi=0$. At $Q^{2}=0$ the relation $\mathcal{H}_{\pi}(x, 0)=q_{\pi}(x)$ follows. We choose the LFWFs $\psi_{\pi}^{(0)}\left(x, \mathbf{k}_{\perp}\right)$ and $\psi_{\pi}^{(1)}\left(x, \mathbf{k}_{\perp}\right)$ as

$$
\begin{aligned}
& \psi_{\pi}^{(0)}\left(x, \mathbf{k}_{\perp}\right)=\frac{4 \pi N_{0}}{\kappa} \frac{\sqrt{\log (1 / x)}}{1-x} \sqrt{f(x) \bar{f}(x)} \exp \left[-\frac{\mathbf{k}_{\perp}^{2}}{2 \kappa^{2}} \frac{\log (1 / x)}{(1-x)^{2}} \bar{f}(x)\right], \\
& \psi_{\pi}^{(1)}\left(x, \mathbf{k}_{\perp}\right)=\frac{4 \pi N_{1}}{\kappa^{2}} \frac{\sqrt{\log ^{3}(1 / x)}}{(1-x)^{2}} \sqrt{f(x) \bar{f}^{3}(x)} \exp \left[-\frac{\mathbf{k}_{\perp}^{2}}{2 \kappa^{2}} \frac{\log (1 / x)}{(1-x)^{2}} \bar{f}(x)\right]
\end{aligned}
$$


Pion light-front wave function, parton distribution and the electromagnetic form factor5

to guarantee the correct scaling behavior of the pion PDF, NPD and form factor due to the contribution of $\psi_{\pi}^{(0)}\left(x, \mathbf{k}_{\perp}\right)$ and $\psi_{\pi}^{(1)}\left(x, \mathbf{k}_{\perp}\right)$. In particular, $\psi_{\pi}^{(0)}\left(x, \mathbf{k}_{\perp}\right)$ produces the following scalings of $q_{\pi}(x) \sim(1-x)^{2}$ and $\mathcal{H}_{\pi}\left(x, Q^{2}\right) \sim(1-x)^{2}$ for large $x \rightarrow 1$ and $F_{\pi}\left(Q^{2}\right) \sim 1 / Q^{2}$ for large $Q^{2} \rightarrow \infty$. The LFWF $\psi_{\pi}^{(1)}\left(x, \mathbf{k}_{\perp}\right)$ results in $q_{\pi}(x) \sim(1-x)^{5}$, $\mathcal{H}_{\pi}\left(x, Q^{2}\right) \sim(1-x)^{5}$ for $x \rightarrow 1$ and $F_{\pi}\left(Q^{2}\right) \sim 1 / Q^{4}$ for $Q^{2} \rightarrow \infty$. The functions $f(x)$ and $\bar{f}(x)$ are specified as

$$
f(x)=x^{\alpha-1}(1-x)^{\beta}\left(1+\gamma x^{\delta}\right), \quad \bar{f}(x)=x^{\bar{\alpha}}(1-x)^{\beta}\left(1+\bar{\gamma} x^{\bar{\delta}}\right) .
$$

The $\alpha, \beta, \gamma, \delta, \bar{\alpha}, \bar{\gamma}$ and $\bar{\delta}$ are free parameters and $N_{0}$ and $N_{1}$ are the normalization factors.

A straightforward calculation gives

$$
\begin{aligned}
\mathcal{H}_{\pi}\left(x, Q^{2}\right) & =N_{0}^{2} f(x) \exp \left[-\frac{Q^{2}}{4 \kappa^{2}} \log (1 / x) \bar{f}(x)\right] \\
& \times\left[1+\left(\frac{N_{1}}{N_{0}}\right)^{2} \bar{f}(x) \log (1 / x)\left(1-\frac{Q^{2}}{4 \kappa^{2}} \log (1 / x) \bar{f}(x)\right)\right] .
\end{aligned}
$$

The parameter $N_{0}$ is fixed from the normalization condition (11), while the ratio $N_{1} / N_{0}$ is a free parameter.

The function $f(x)$ is constrained by the pion PDF fixed through Ref. [7]

$$
q_{\pi}(x) \sim f(x),
$$

while the function $\bar{f}(x)$ is fixed from the analysis of the pion electromagnetic form factor (as will be shown below). Note that the improved LFWF $\psi_{\pi}\left(x, \mathbf{k}_{\perp}\right)$ reduces to the AdS/QCD LFWF $\psi_{\pi}^{\operatorname{AdS}}\left(x, \mathbf{k}_{\perp}\right)$ in the limit $f(x)=\bar{f}(x)=1$. By construction the functions $f(x)$ and $\bar{f}(x)$ have the same scaling behavior of $(1-x)^{\beta}$ at large $x$ to guarantee the correct $1 / Q^{2}$ power scaling of the pion form factor at large $Q^{2}$ independent on the value of $\beta$ (see below).

Next we discuss the choice of the free parameters $\alpha, \beta, \gamma, \bar{\alpha}$ and $\bar{\gamma}$. The values for $\alpha, \beta, \gamma$ (central values) are taken from Ref. [7] (see Eq. (2)). The parameters $\bar{\alpha}, \bar{\gamma}$ and $\bar{\delta}$ are fixed by a fit to the pion electromagnetic form factor resulting in:

$$
\bar{\alpha}=0.15, \bar{\gamma}=2.5, \bar{\delta}=2 \text {. }
$$

Also we use $N_{1} / N_{0}=0.3$. As stressed before, the pion form factor has the correct $1 / Q^{2}$ scaling for large $Q^{2}$, consistent with quark counting rules. It is also independent of $\beta$, which governs the power scaling $(1-x)^{\beta}$ of the pion PDF and GPD at large $x$. Here we use $\beta=2.03$ extracted from the updated analysis of the E615 experimental data [8] including NLL threshold resummation effects [7]. The choice of our parameters is constrained by data on the electromagnetic radius of the pion with $r_{\pi}^{\exp }=0.672 \pm 0.008$ $\mathrm{fm}$ [26]. In particular, we are able to reproduce the central value of $r_{\pi}=0.672 \mathrm{fm}$. In Fig. 1 we show our result for the pion valence PDF, indicating the leading contribution due to $L_{z}=0$ LFWF (thick line), which fits the result of Ref. [7], and the total result including both $L_{z}=0$ and $\left|L_{z}\right|=1$ LWFs (dashed line). The total result can reproduce the result of Ref. [7] for the pion PDF, when two of our parameters are slightly changed $(\alpha=0.70 \rightarrow 0.75$ and $\bar{\delta}=2 \rightarrow 1.65)$. In Fig. 2 we show our result for the pion 
Pion light-front wave function, parton distribution and the electromagnetic form factor6

electromagnetic form factor multiplied by $Q^{2}$ and compare it to data. In Fig. 3 we show a three-dimensional plot of the pion NPD as function of $x$ in the interval from 0 to 1 and $Q^{2}$ from 0 to $10 \mathrm{GeV}^{2}$.

In conclusion, we state again the main result of this report. We first take into account the result of Ref. [7], which produces a considerably softer pion PDF when the NLL threshold resummation effects in the cross section of $\pi^{-} N \rightarrow \mu^{+} \mu^{-} X$ process are included. We derive an improved pion LFWF at this order of accuracy. This LFWF reproduces several fundamental properties of the pion consistent with data - valence parton distribution, electromagnetic form factor and radius. The derived LFWF is parametrized by two functions $f(x)$ and $\bar{f}(x)$ depending on the light-cone variable $x$, which have a clear physical interpretation. In particular, the function $f(x)$ is related to the pionic PDF at the initial scale derived in Ref. [7]. The function $\bar{f}(x)$ defines the $Q^{2}$ dependence of the pion GPD and the electromagnetic form factor. Both functions $f(x)$ and $\bar{f}(x)$ have exactly the same power scaling $(1-x)^{\beta}$ at large $x \rightarrow 1$. This behavior provides the correct power scaling of the pion form factor. In particular, the $L_{z}=0$ $\operatorname{LFWF} \psi^{(0)}\left(x, \mathbf{k}_{\perp}\right)$ and $\left|L_{z}\right|=1 \operatorname{LFWF} \psi^{(1)}\left(x, \mathbf{k}_{\perp}\right)$ give the following scaling of the pion form factor at large $Q^{2} \rightarrow \infty$

$$
\begin{aligned}
& F_{\pi}^{(0)}\left(Q^{2}\right) \sim \int_{0}^{1} d x(1-x)^{\beta} \exp \left[-\frac{Q^{2}}{4 \kappa^{2}}(1-x)^{\beta+1}\right] \sim \frac{1}{Q^{2}}, \\
& F_{\pi}^{(1)}\left(Q^{2}\right) \sim \int_{0}^{1} d x(1-x)^{2 \beta+1} \exp \left[-\frac{Q^{2}}{4 \kappa^{2}}(1-x)^{\beta+1}\right] \sim \frac{1}{Q^{4}},
\end{aligned}
$$

respectively, which are consistent with quark counting rules. Using data on the pion electromagnetic form factor we constrain the form of the function $\bar{f}(x)$ and also produce the result for the pion nonforward parton density $\mathcal{H}_{\pi}\left(x, Q^{2}\right)$ at zero skewness.

\section{Acknowledgments}

The authors thank Werner Vogelsang for useful discussions. This work was supported by the DFG under Contract No. LY 114/2-1, by the German Bundesministerium für Bildung und Forschung (BMBF) under Grant No. 05P12VTCTG, by Marie Curie Reintegration Grant IRG 256574, by CONICYT (Chile) Research Project No. 80140097, by CONICYT (Chile) under Grant No. 7912010025, by FONDECYT (Chile) under Grant No. 1140390 and No. 1141280, by Tomsk State University Competitiveness Improvement Program and the Russian Federation program "Nauka" (Contract No. 0.1526.2015, 3854). V.E.L. would like to thank Departamento de Física

y Centro Científico Tecnológico de Valparaíso (CCTVal), Universidad Técnica Federico Santa María, Valparaíso, Chile and Instituto de Física y Astronomía, Universidad de Valparaíso, Chile for warm hospitality. 
Pion light-front wave function, parton distribution and the electromagnetic form factor 7

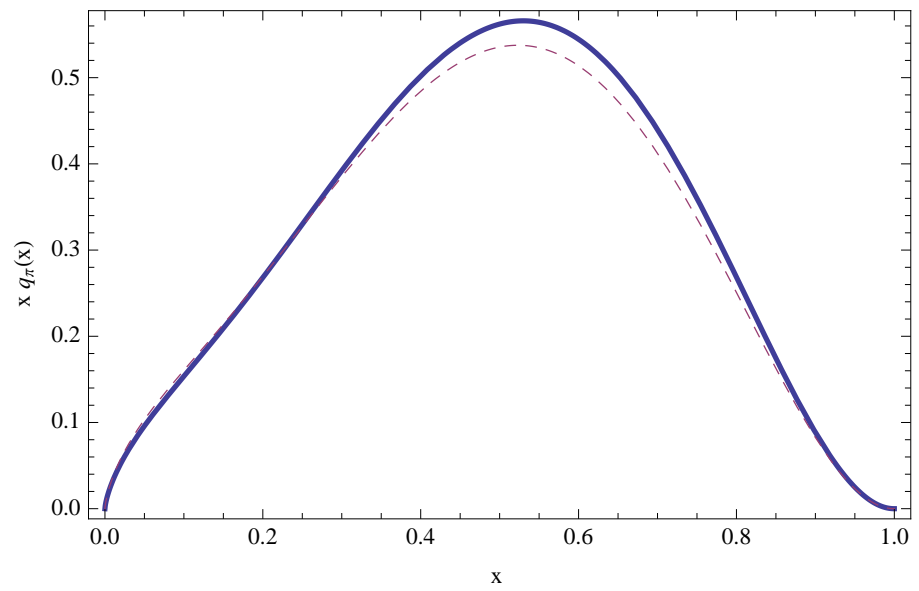

Figure 1. Pion valence PDF $x q_{\pi}(x)$. The thick line corresponds to the leading $L_{z}=0$ LFWF contribution, while the dashed line includes both $L_{z}=0$ and $\left|L_{z}\right|=1$ LFWF contributions.

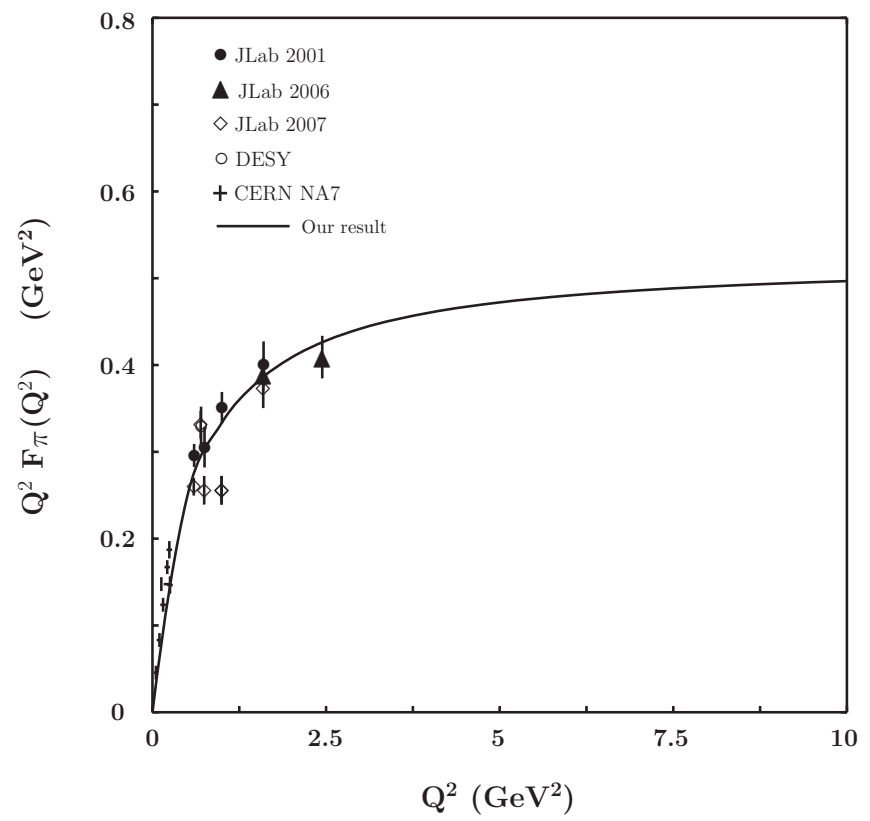

Figure 2. Pion form factor $Q^{2} F_{\pi}\left(Q^{2}\right)$. Data taken from [1]-[6]. 
Pion light-front wave function, parton distribution and the electromagnetic form factor 8

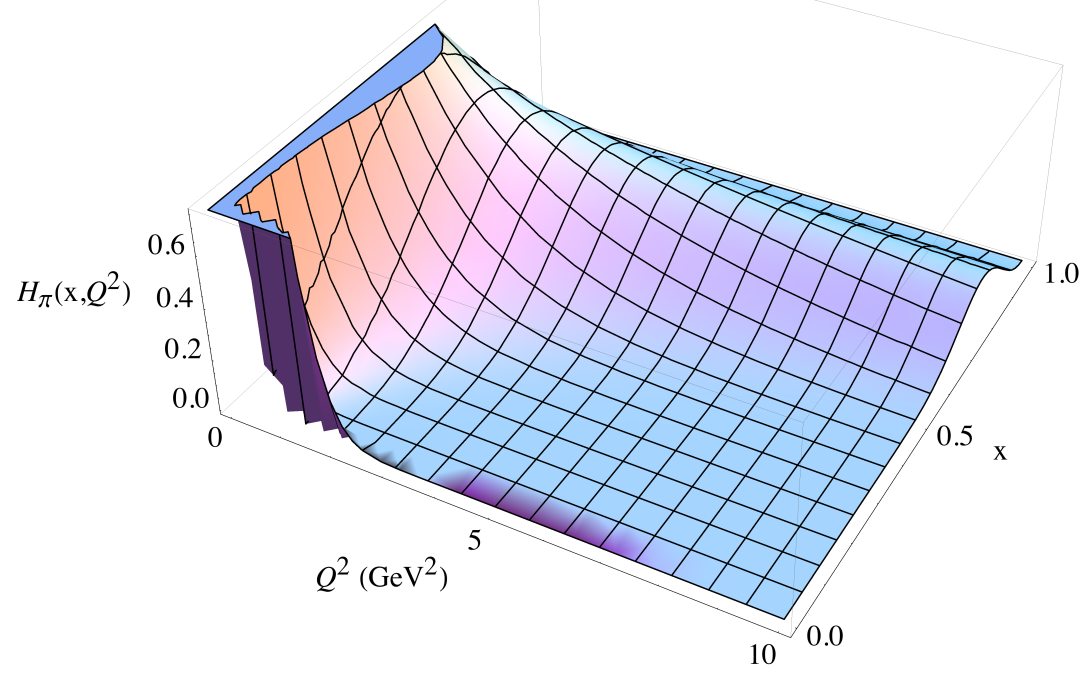

Figure 3. Pion nonforward parton density $\mathcal{H}_{\pi}\left(x, Q^{2}\right)$ for $x=[0,1]$ and $Q^{2}=\left[0,10 \mathrm{GeV}^{2}\right]$.

\section{Appendix A. Quark-antiquark Fock states and electromagnetic transition operator in light-front frame}

Here we specify quark-antiquark Fock states and electromagnetic transition operator in terms of creation and annihilation operators in the light-front formalism. The $u \bar{d}$ Fock states with specific value of $L_{z}=0, \pm 1$ are

$$
\begin{aligned}
& \left|x, \mathbf{k}_{\perp} ; P^{+}, \mathbf{P}_{\perp}, L_{z}=0\right\rangle=\frac{1}{\sqrt{6}} \sum_{a=1}^{3}\left(b_{u \uparrow}^{\dagger a}(1) d_{d \downarrow}^{\dagger a}(2)-b_{u \downarrow}^{\dagger a}(1) d_{d \uparrow}^{\dagger a}(2)\right)|0\rangle, \\
& \left|x, \mathbf{k}_{\perp} ; P^{+}, \mathbf{P}_{\perp}, L_{z}=-1\right\rangle=\frac{1}{\sqrt{3}} \sum_{a=1}^{3} b_{u \uparrow}^{\dagger a}(1) d_{d \uparrow}^{\dagger a}(2)|0\rangle \\
& \left|x, \mathbf{k}_{\perp} ; P^{+}, \mathbf{P}_{\perp}, L_{z}=1\right\rangle=\frac{1}{\sqrt{3}} \sum_{a=1}^{3} b_{u \downarrow}^{\dagger a}(1) d_{d \downarrow}^{\dagger a}(2)|0\rangle
\end{aligned}
$$

where $1 \doteq x P^{+}, \mathbf{k}_{\perp}+x \mathbf{P}_{\perp}$ and $2 \doteq(1-x) P^{+},-\mathbf{k}_{\perp}+(1-x) \mathbf{P}_{\perp}$. Here $b_{\lambda}^{\dagger a}\left(b_{\lambda}^{a}\right)$ and $d_{\lambda}^{\dagger a}\left(d_{\lambda}^{a}\right)$ are the creation (annihilation) operators of $u$-quark and $\bar{d}$ quark with color $a$, respectively, obeying the nonvanishing anticommutation relations

$$
\begin{aligned}
& \left\{b_{\lambda}^{a}\left(k^{+}, \mathbf{k}_{\perp}\right), b_{\lambda^{\prime}}^{\dagger a^{\prime}}\left(k^{+^{\prime}}, \mathbf{k}_{\perp}^{\prime}\right)\right\}=\left\{d_{\lambda}^{a}\left(k^{+}, \mathbf{k}_{\perp}\right), d_{\lambda^{\prime}}^{\dagger a^{\prime}}\left(k^{+^{\prime}}, \mathbf{k}_{\perp}^{\prime}\right)\right\} \\
= & 2 k^{+} \delta_{\lambda \lambda^{\prime}} \delta^{a a^{\prime}} \delta\left(k^{+}-k^{+^{\prime}}\right) \delta^{2}\left(\mathbf{k}_{\perp}-\mathbf{k}_{\perp}^{\prime}\right) .
\end{aligned}
$$

The electromagnetic current transition operator in terms of creation and annihilation operators of $u$ and $\bar{d}$ quarks read as [15]

$$
\begin{aligned}
J^{+}(0) & =\int \frac{d q^{+} d^{2} \mathbf{q}_{\perp}}{(2 \pi)^{3} \sqrt{2 q^{+}}} \int \frac{d q^{\prime}+d^{2} \mathbf{q}_{\perp}^{\prime}}{(2 \pi)^{3} \sqrt{2 q^{\prime}+}} \sum_{a=1}^{3}\left[e_{u} b_{u \uparrow}^{\dagger a}(q) b_{u \uparrow}^{a}\left(q^{\prime}\right)+e_{u} b_{u \downarrow}^{\dagger a}(q) b_{u \downarrow}^{a}\left(q^{\prime}\right)\right. \\
& \left.+e_{\bar{d}} d_{d \uparrow}^{\dagger a}(q) d_{d \uparrow}^{a}\left(q^{\prime}\right)+e_{\bar{d}} d_{d \downarrow}^{\dagger a}(q) d_{d \downarrow}^{a}\left(q^{\prime}\right)\right]
\end{aligned}
$$


Pion light-front wave function, parton distribution and the electromagnetic form factor 9

[1] J. Volmer et al. (The Jefferson $F_{\pi}$ Collaboration), Phys. Rev. Lett. 86, 1713 (2001).

[2] C. J. Bebek, Phys. Rev. D 17, 1693 (1978).

[3] P. Brauel et al., Z. Phys. C 3, 101 (1979).

[4] S. R. Amendolia et al. (CERN NA7 Collaboration), Nucl. Phys. B 277, 168 (1986).

[5] T. Horn et al. (Jefferson Lab F(pi)-2 Collaboration), Phys. Rev. Lett. 97, 192001 (2006).

[6] V. Tadevosyan et al. (Jefferson Lab F(pi) Collaboration), Phys. Rev. C 75, 055205 (2007).

[7] M. Aicher, A. Schafer and W. Vogelsang, Phys. Rev. Lett. 105, 252003 (2010).

[8] J. S. Conway, C. E. Adolphsen, J. P. Alexander, K. J. Anderson, J. G. Heinrich, J. E. Pilcher, A. Possoz and E. I. Rosenberg et al., Phys. Rev. D 39, 92 (1989).

[9] P. J. Sutton, A. D. Martin, R. G. Roberts and W. J. Stirling, Phys. Rev. D 45, 2349 (1992).

[10] M. Gluck, E. Reya and I. Schienbein, Eur. Phys. J. C 10, 313 (1999).

[11] K. Wijesooriya, P. E. Reimer and R. J. Holt, Phys. Rev. C 72, 065203 (2005).

[12] M. B. Hecht, C. D. Roberts and S. M. Schmidt, Phys. Rev. C 63, 025213 (2001).

[13] W. Broniowski, E. Ruiz Arriola and K. Golec-Biernat, Phys. Rev. D 77, 034023 (2008).

[14] T. Gutsche, V. E. Lyubovitskij, I. Schmidt and A. Vega, Phys. Rev. D 89, 054033 (2014).

[15] S. J. Brodsky, G. F. de Teramond, Phys. Rev. D 77, 056007 (2008).

[16] S. J. Brodsky, F. -G. Cao and G. F. de Teramond, Phys. Rev. D 84, 075012 (2011).

[17] A. Vega, I. Schmidt, T. Branz, T. Gutsche and V. E. Lyubovitskij, Phys. Rev. D 80, 055014 (2009).

[18] T. Branz, T. Gutsche, V. E. Lyubovitskij, I. Schmidt and A. Vega, Phys. Rev. D 82, 074022 (2010).

[19] T. Gutsche, V. E. Lyubovitskij, I. Schmidt and A. Vega, Phys. Rev. D 87, 056001 (2013).

[20] A. Vega, I. Schmidt, T. Gutsche and V. E. Lyubovitskij, Phys. Rev. D 83, 036001 (2011); T. Gutsche, V. E. Lyubovitskij, I. Schmidt and A. Vega, Phys. Rev. D 85, 076003 (2012);

T. Gutsche, V. E. Lyubovitskij, I. Schmidt and A. Vega, Phys. Rev. D 86, 036007 (2012); Phys.

Rev. D 87, 016017 (2013).

[21] M. Burkardt, X. D. Ji and F. Yuan, Phys. Lett. B 545, 345 (2002). [22]

[22] X. D. Ji, J. P. Ma and F. Yuan, Eur. Phys. J. C 33, 75 (2004).

[23] B. Pasquini and P. Schweitzer, Phys. Rev. D 90, 014050 (2014).

[24] A. V. Radyushkin, Phys. Rev. D 58, 114008 (1998).

[25] S. D. Drell and T. -M. Yan, Phys. Rev. Lett. 24, 181 (1970).

[26] K. A. Olive et al. (Particle Data Group Collaboration), Chin. Phys. C 38, 090001 (2014). 